\section{TEN YEARS OF THE MONASH LUPUS CLINIC AND ONWARDS - MODEL OF A MULTIDISCIPLINARY SPECIALIST CLINIC PROVIDING IMPROVED HEALTHCARE QUALITY AND OUTCOMES FOR SLE PATIENTS}

${ }^{1} \mathrm{~S}$ Toor, ${ }^{1} \mathrm{R}$ Koelmeyer, ${ }^{1,2} \mathrm{~V}$ Golder, ${ }^{1,2} \mathrm{E}$ Morand, ${ }^{1,2}{ }^{2} \mathrm{~A} \mathrm{Hoi}{ }^{*} .{ }^{1}$ Monash University - School of Clinical Sciences, Faculty of Medicine- Nursing and Health Sciences, Clayton, Australia; ${ }^{2}$ Monash Health, Rheumatology, Clayton, Australia

10.1136/lupus-2017-000215.440

Background and aims The Monash Lupus Clinic is Australia's first multi-disciplinary specialist lupus clinic, which runs in parallel with a clinical registry program providing healthcare quality and outcomes data for research purposes. We aim to provide an overview of the characteristics and longitudinal outcomes of lupus patients treated at the Monash Lupus Clinic over the last ten years.

Methods Outcome measures included disease activity, medication use, damage accrual and other clinically-relevant events.

Results Over the last 10 years, we have observed improvements in indices associated with better healthcare quality. We observed a significant reduction in the proportion of patients with a maximum SLEDAI- $2 \mathrm{~K}$ score $\geq 10$ over time (chi ${ }^{2}$ test for trend, $\mathrm{p}<0.001$ ), and a significant trend of an increase in the proportion of patients spending $>50 \%$ of their observed time meeting the criteria for LLDAS $\left(\mathrm{chi}^{2}\right.$ test for trend, $p=0.005)$. There was also a reduction in the proportion of patients experiencing at least one mild/moderate flare $(\mathrm{p}<0.001)$ or severe flare $(\mathrm{p}=0.006)$. We also observed a change in the medication use over the last 10 years, with a notable reduction in the use of higher doses of prednisolone (>7.5 mg/day; $\mathrm{p}=0.014)$.

Conclusions Running a multi-disciplinary clinic alongside research activity is both feasible and worthwhile. Systematic collection of longitudinal data on SLE patients has shown changes that reflect better control of disease. As more targeted therapies become available for the treatment of lupus, we expect that such registry data will provide valuable real world evidence of the effectiveness of treatments and management strategies.

\section{USE OF A PATIENT-REPORTED OUTCOME MEASURE, THE LUPUSQOL TO REFER PATIENTS FOR FURTHER SUPPORT - A PRELIMINARY STUDY}

V Kakkar, K McElhone, LS Teh*. Royal Blackburn Hospital, Department Of Rheumatology, Blackburn, UK

\subsection{6/lupus-2017-000215.441}

Background and aims Systemic Lupus Erythematosus (SLE) is a multisystem disease and patients may benefit from non-pharmacological interventions. We explored the utility of the LupusQoL in identifying patients who may benefit from physical, occupational or psychological support.

Methods The LupusQoL is a disease-specific health-related quality of life (HRQoL) questionnaire with eight domains (physical health, pain, planning, intimate relationship, burden to others, emotional health, body image and fatigue). For each domain the scores range from 0 to 100 (higher score better health). SLE patients sequentially attending an outpatient clinic over a 6 month period, who were able to complete the questionnaire in English, were asked to complete the questionnaire. Patients who scored $<50$ were offered physical,
Abstract 441 Table 1

\begin{tabular}{|c|c|c|c|c|}
\hline Patients & $\begin{array}{c}\text { Any Domain } \\
\text { score }<50 \\
\mathrm{n}=14\end{array}$ & Physiotherapy & Occupational & Psychology \\
\hline Referred & & 10 & 10 & 6 \\
\hline Attended & & 10 & 10 & 5 \\
\hline
\end{tabular}

occupational and/or psychological support dependent on the domains affected.

Results 40 patients $(97.5 \%$ females, $75 \%$ Caucasians; $17.5 \%$ South-Asians; 5\% Afro-Caribbean; mean age 52.2 years) completed the LupusQoL over 6 months. Table 1 shows the number of patients who were eligible, the numbers who were referred and the numbers who attended the different support services. All domains of the LupusQol were impaired, with fatigue (45.19) being the worst affected and emotional health (68.60) being the least.

Conclusions Following attendance for physical, occupational and psychological support, we intend to re-administer the LupusQoL to determine if these non-pharmacological interventions have improved HRQoL. The appropriateness of the LupusQoL threshold used to identify the intervention need will also be evaluated.

\section{THE HIGH DISEASE ACTIVITY STATE IS AN ADVERSE PROGNOSTIC INDICATOR IN SLE AND DEFINES A CLINICALLY DISTINCT POPULATION}

R Koelmeyer, E Morand, A Hoi*. School of Clinical Sciences at Monash Health, Centre for Inflammatory Diseases, Clayton, Australia

\subsection{6/lupus-2017-000215.442}

Background and aims To identify whether a SLEDAI-2K score $\geq 10$, herein termed the High Disease Activity State (HDAS), might have utility as a prognostic factor amongst patients with Systemic Lupus Erythematosus (SLE).

Methods Using clinical data collected via the Monash Lupus Clinic we assessed the association of ever experiencing HDAS with adverse clinical outcomes and the association of sociodemographic and disease characteristics with the odds of ever experiencing HDAS. Logistic regression or generalised estimating equations were used for the analyses. For analyses of longitudinal outcomes, associations were adjusted for observation time.

Results Overall, 211 patients meeting SLE classification criteria were observed for a median of 4.5 years (range: 1-7.9 years); 42.7\% experienced HDAS at least once during this time. The median time to first HDAS was 9 months after enrolment (range 0-6.7 years). Being diagnosed with SLE at $\geq 45$ years of age was associated with reduced odds of experiencing HDAS amongst female patients $(\mathrm{p}=0.004)$. Autoantibody-positivity for anti-dsDNA was strongly associated with increased odds of ever experiencing HDAS $(\mathrm{p}<0.001)$. Ever experiencing HDAS was associated with increased odds of having an Adjusted Mean SLEDAI score in the highest quartile, experiencing a greater number of flares and of accruing damage (overall and particularly renal damage) over the observation period ( $\mathrm{p} \leq 0.003$ for all). Patients ever experiencing HDAS were also more likely to experience neuropsychiatric, renal 